\title{
VERSATILE ACTIVE BIQUAD BASED ON DUAL-OUTPUT SECOND-GENERATION CURRENT CONVEYORS
}

\author{
M. T. ABUELMA'ATTI and H. A. ALZAHER
}

King Fahd University of Petroleum and Minerals, Box 203, Dhahran 31261, Saudi Arabia

(Received 16 March 1997; In final form 22 July 1997)

\begin{abstract}
A universal active biquad based on the dual-output second-generation current-conveyor (CCII \pm ) is presented. The circuit uses only six CCII \pm and can, simultaneously, support three mixed-mode transfer functions and five current-mode transfer functions. The circuit uses grounded resistors and capacitors and enjoys independent grounded-element control of the filter parameters.
\end{abstract}

Keywords: Active filters; current conveyors

\section{INTRODUCTION}

In a recent publication, Sun and Fidler [1], present a versatile active biquad using seven second-generation current-conveyors. The circuit can simultaneously realize four different filter characteristics; one output current and three output voltages. The output current can realize low-pass, highpass, bandpass and bandstop characteristics. Realization of allpass characteristics is feasible, but requires replacing a plus-type second-generation current-conveyor (CCII + ) by a minustype second-generation current-conveyor (CCII-). The output voltages can simultaneously realize lowpass, highpass and bandpass characteristics. 
Here we propose to use the dual-output CCII士; its symbolic representation and matrix equation are shown in Figure 1, for realizing single-input multiple-output active biquad. The proposed circuit can simultaneously support eight different current-mode and mixed-mode transfer functions, while using only six dual-output CCII \pm .

\section{Proposed Circuit}

The proposed circuit, using only $\mathrm{CCII} \pm$, is as shown in Figure 2. Assuming ideal CCII \pm , with $v_{y}=v_{x}, i_{y}=0, i_{z_{ \pm}}= \pm i_{x}$, routine analysis shows that:

$$
\begin{aligned}
& \frac{V_{1}}{I_{i}}=\frac{s^{2} C_{1} C_{2}}{s^{2} C_{1} C_{2} G_{b 2}+s C_{1} G_{2} G_{b 1}+G_{1} G_{2} G_{b 0}} \\
& \frac{V_{2}}{I_{i}}=\frac{s C_{1} G_{2}}{s^{2} C_{1} C_{2} G_{b 2}+s C_{1} G_{2} G_{b 1}+G_{1} G_{2} G_{b 0}}
\end{aligned}
$$

$$
\frac{V_{3}}{I_{i}}=\frac{G_{1} G_{2}}{s^{2} C_{1} C_{2} G_{b 2}+s C_{1} G_{2} G_{b 1}+G_{1} G_{2} G_{b 0}}
$$

$$
\frac{I_{01}}{I_{i}}=-\frac{G_{2} V_{1}}{I_{i}}=-\frac{s^{2} C_{1} C_{2} G_{2}}{s^{2} C_{1} C_{2} G_{b 2}+s C_{1} G_{2} G_{b 1}+G_{1} G_{2} G_{b 0}}
$$

$$
\frac{I_{02}}{I_{i}}=-\frac{G_{1} V_{2}}{I_{i}}=-\frac{s C_{1} G_{1} G_{2}}{s^{2} C_{1} C_{2} G_{b 2}+s C_{1} G_{2} G_{b 1}+G_{1} G_{2} G_{b 0}}
$$

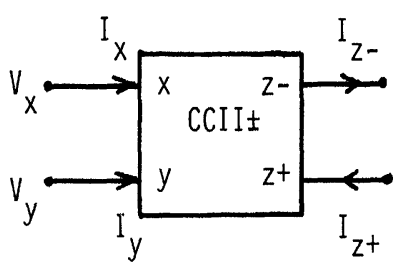

(a)

$$
\left[\begin{array}{l}
V_{x} \\
I_{y} \\
I_{z-} \\
I_{z+}
\end{array}\right]=\left[\begin{array}{cccc}
0 & +1 & 0 & 0 \\
0 & 0 & 0 & 0 \\
+1 & 0 & 0 & 0 \\
+1 & 0 & 0 & 0
\end{array}\right]\left[\begin{array}{c}
I_{x} \\
V_{y} \\
V_{z-} \\
V_{z+}
\end{array}\right]
$$

(b)

FIGURE 1 (a) Symbolic representation and (b) matrix of the CCII \pm . 


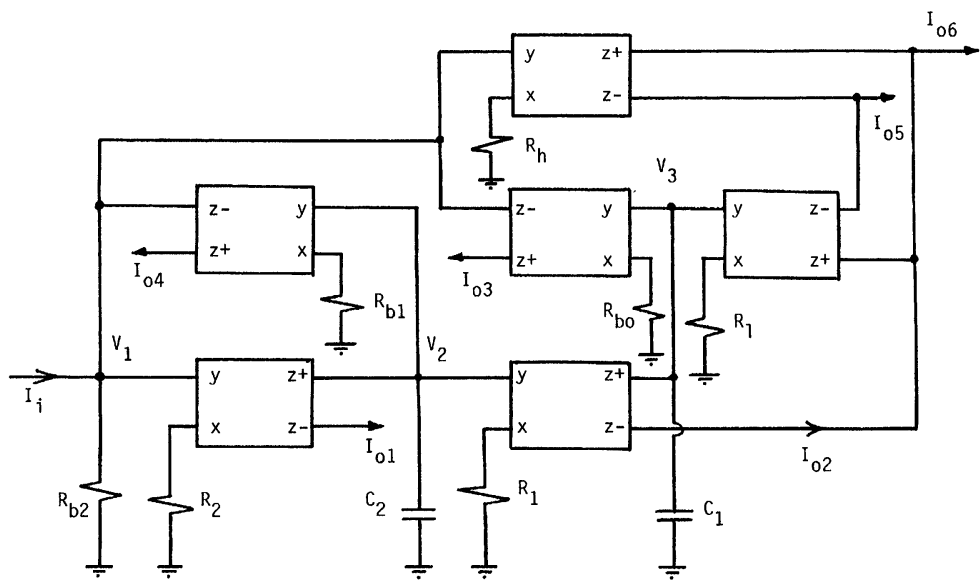

FIGURE 2 Proposed Versatile biquad based on dual-output CCII \pm .

$$
\begin{gathered}
\frac{I_{03}}{I_{i}}=\frac{G_{b 0} V_{3}}{I_{i}}=\frac{G_{b 0} G_{1} G_{2}}{s^{2} C_{1} C_{2} G_{b 2}+s C_{1} G_{2} G_{b 1}+G_{1} G_{2} G_{b 0}} \\
\frac{I_{04}}{I_{i}}=\frac{G_{b 1} V_{2}}{I_{i}}=\frac{s C_{1} G_{2} G_{b 1}}{s^{2} C_{1} C_{2} G_{b 2}+s C_{1} G_{2} G_{b 1}+G_{1} G_{2} G_{b 0}} \\
\frac{I_{05}}{I_{i}}=-\frac{G_{h} V_{1}+G_{l} V_{3}}{I_{i}}=-\frac{s^{2} C_{1} C_{2} G_{h}+G_{1} G_{2} G_{l}}{s^{2} C_{1} C_{2} G_{b 2}+s C_{1} G_{2} G_{b 1}+G_{1} G_{2} G_{b 0}}
\end{gathered}
$$

and

$$
\frac{I_{06}}{I_{i}}=\frac{I_{02}+G_{h} V_{1}+G_{l} V_{3}}{I_{i}}=\frac{s^{2} C_{1} C_{2} G_{h}-s C_{1} G_{1} G_{2}+G_{1} G_{2} G_{l}}{s^{2} C_{1} C_{2} G_{b 2}+s C_{1} G_{2} G_{b 1}+G_{1} G_{2} G_{b 0}}
$$

Equations $(1-3)$ represent the transfer functions of mixed-mode; with input current and output voltage, highpass, bandpass and lowpass filters respectively. Equations (4), (6-8) represent the transfer functions of current-mode; with input current and output current, highpass, lowpass, bandpass, and bandstop filters respectively. With $G_{h}=G_{b 2}, G_{l}=G_{b l}$ and $G_{l}=G_{b 0}$, Eq. (9) represents the transfer function of an allpass filter. Thus the circuit of Figure 2 can, 
simultaneously, support eight different current-mode and mixed-mode transfer functions.

Compared to the circuit proposed by Sun and Fidler [1], the proposed circuit have the following advantages:

1) Use of fewer number of active elements; only six CCII \pm rather than seven as proposed in [1].

2) Simultaneous support of eight different transfer functions; three mixed-mode and five current-mode. The circuit proposed in [1] can simultaneously support only three voltage-mode and one currentmode transfer functions.

From $(1-9)$ it can be seen that the parameters $\omega_{0}$ and $\omega_{0} / Q_{0}$ expressed as

$$
\omega_{0}^{2}=\frac{G_{1} G_{2} G_{b 0}}{C_{1} C_{2} G_{b 2}}
$$

and

$$
\frac{\omega_{0}}{Q_{0}}=\frac{G_{2} G_{b 1}}{C_{2} G_{b 2}}
$$

From (10) and (11) it can be seen that the paramter $\omega_{0}$ can be controlled by adjusting the resistance $R_{1}=\left(1 / G_{1}\right)$ and/or the resistance $R_{b 0}=\left(1 / G_{b 0}\right)$ and/or the capacitor $C_{1}$ without disturbing the parameter $\omega_{0} / Q_{0}$. It can also be seen that the parameter $\omega_{0} / Q_{0}$ can be controlled by adjusting the resistance $R_{b 1}=\left(1 / G_{b 1}\right)$ without disturbing the parameter $\omega_{0}$. Thus the circuit enjoys independent grounded-element control of the parameters $\omega_{0}$ and $\omega_{0} / Q_{0}$. However, for the allpass realisaiton, and because of the required matching conditions, the parameter $\omega_{0}$ can be controlled only by adjusting the capacitance $C_{1}$ without disturbing the parameter $\omega_{0} / Q_{0}$. The parameter $\omega_{0} / Q_{0}$ cannot be controlled without disturbing the parameter $\omega_{0}$.

\section{CONCLUSION}

A new active biquad based on dual-output second-generation current conveyors has been presented. The proposed circuit uses only six 
$\mathrm{CCII} \pm$ and can simultaneously support eight different filter transfer function. Compared to the circuit proposed in [1], the present circuit uses less number of active components and can simultaneously provide larger number of different transfer functions while using grounded capacitors and resistors. Similar to the circuit proposed in [1], the present circuit enjoys independent grounded-element control of the filter parameters. These features make the circuit more attractive for integration.

\section{References}

[1] Sun, Y. and Fidler, J. K. (1994). Versatile active biquad based on second-generation current conveyors, International Journal of Electronics, 76, 91-98. 

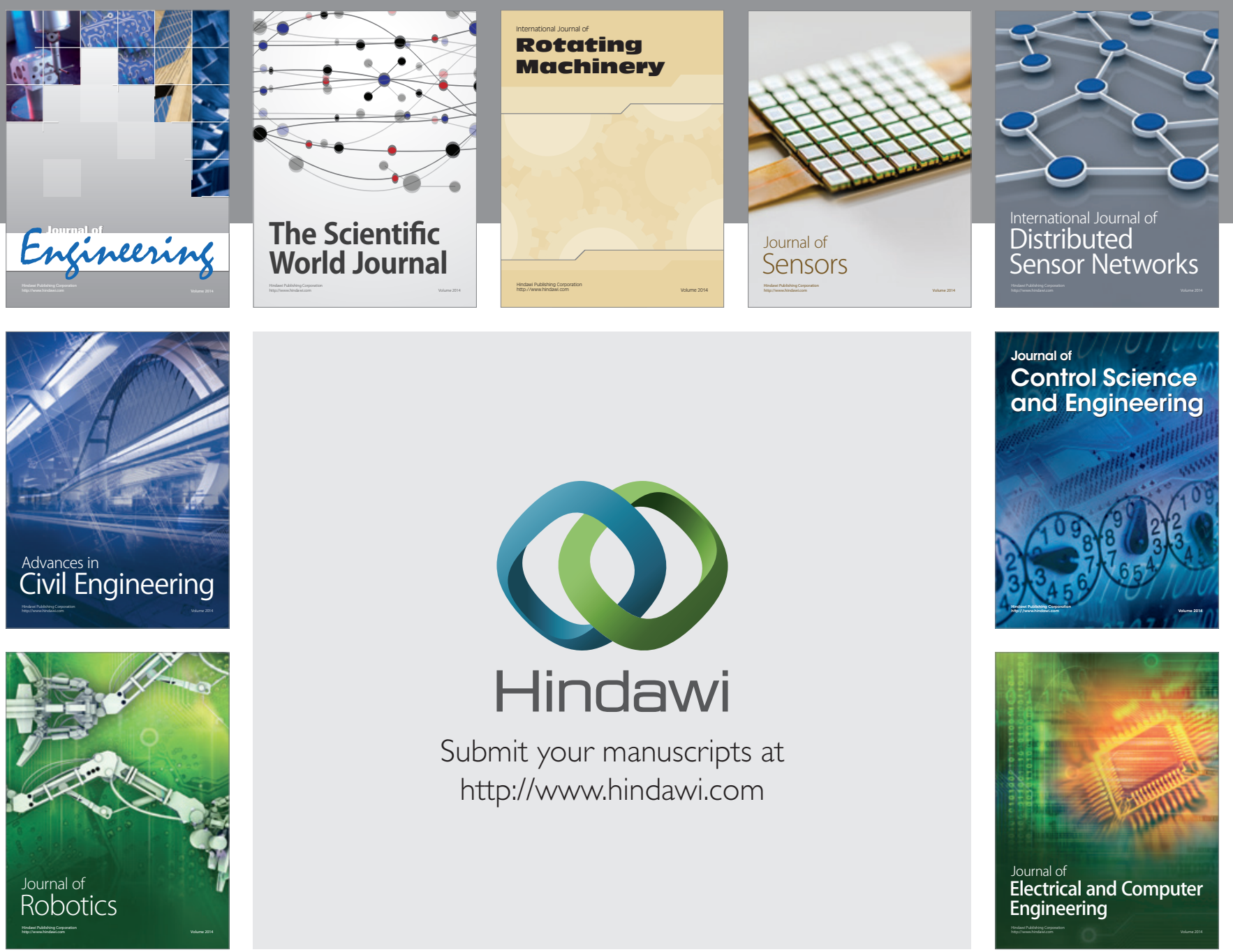

Submit your manuscripts at

http://www.hindawi.com
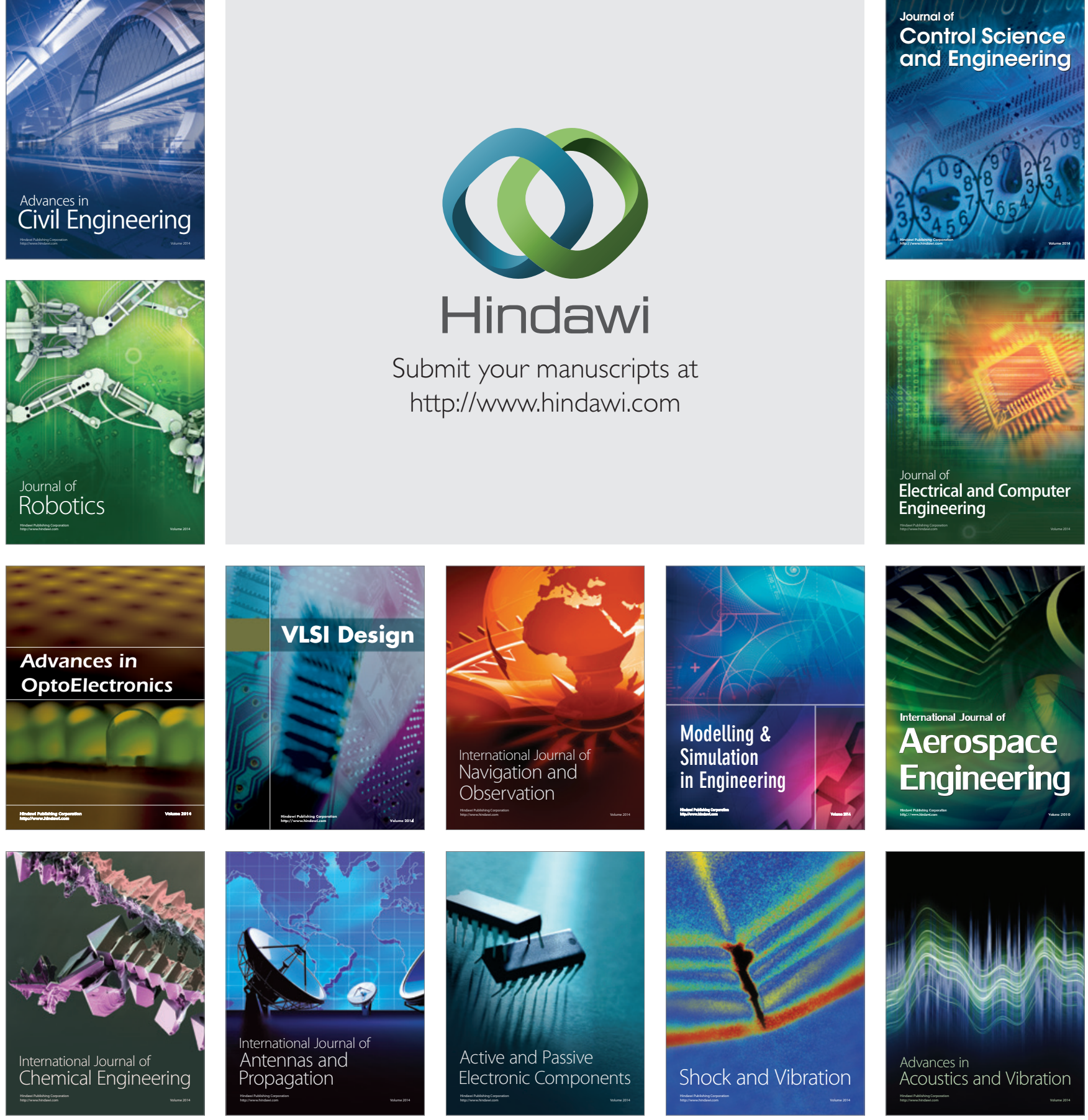